\title{
Original
}

\section{Micro- and Nanostructural Characteristics of Rat Masseter Muscle Entheses}

\author{
Keitaro Arakawa $^{1,2)}$, Satoru Matsunaga ${ }^{2,3)}$, Kunihiko Nojima ${ }^{1,2)}$, Takayoshi Nakano ${ }^{4)}$, \\ Shinichi Abe ${ }^{3)}$, Masao Yoshinari ${ }^{2)}$ and Kenji Sueishi ${ }^{1,2)}$ \\ ${ }^{1)}$ Department of Orthodontics, Tokyo Dental College, Tokyo, Japan \\ 2) Oral Health Science Center, Tokyo Dental College, Tokyo, Japan \\ 3) Department of Anatomy, Tokyo Dental College, Tokyo, Japan \\ 4) Division of Materials \& Manufacturing Science, Graduate School of Engineering, Osaka University, Osaka, Japan \\ (Accepted for publication, September 28, 2019)
}

\begin{abstract}
The entheses of the masticatory muscles differ slightly from those of the trunk and limb muscles. However, the bones of the skull are subject to various functional pressures, including masticatory force, resulting in a complex relationship between bone structure and muscle function that remains to be fully elucidated. The present study aimed to clarify aspects of masseter muscle-tendon-bone morphological characteristics and local load environment through quantitative analysis of biological apatite (BAp) crystallite alignment and collagen fiber orientation together with histological examination of the entheses. Result of histological observation, the present findings show that, in the entheses of the masseter muscle in the first molar region, tendon attaches to bone via unmineralized fibrocartilage, while some tendon collagen fibers insert directly into the bone, running parallel to the muscle fibers. Furthermore, BAp crystallites in the same region show uniaxial preferential alignment at an angle that matches the insertion angle of the tendon fibers. Conversely, in the entheses of the masseter muscle in the third molar region, the tendon attaches to the bone via a layer of thickened periosteum and chondrocytes. As in the first molar region, the results of bone quality analysis in the third molar region showed BAp crystallite alignment parallel to the orientation of the tendon fibers. This indicates that the local mechanical environment generates differences in enthesis morphology. The present study showed a greater degree of uniaxial BAp crystallite alignment in entheses with direct insertion rather than indirect tendon-bone attachment and the direction of alignment was parallel to the orientation of tendon fibers. These findings suggest that functional pressure from the masseter muscle greatly affects bone quality as well as the morphological characteristics of the enthesis, specifically causing micro- and nanostructural anisotropy in the direction of resistance to the applied pressure.
\end{abstract}

Key words: Collagen fiber, Biological apatite (BAp) crystallite, Bone quality, Microbeam X-ray diffraction, SHG imaging, Entheses

\section{Introduction}

The tendons of the trunk and limb muscles attach to bones via enthe$\operatorname{ses}^{1-3)}$. These attachment sites are broadly categorized as either fibrous entheses, composed of perforating mineralized collagen fibers, or fibrocartilaginous entheses, comprising a multitissue interface involving the following four tissues of tendon, unmineralized fibrocartilage, mineralized fibrocartilage, and bone ${ }^{4)}$. The composition, structure, and mechanical properties of these multitissue interfaces vary widely, creating spatial gradients that mediate load transfer between soft and hard tissues and minimize stress concentration ${ }^{5}$. Muscle loading is extremely important for healthy enthesis formation and suppression of muscle function greatly diminishes the biomechanical performance of the enthesis ${ }^{6}$.

Histological examination by Hems et al. revealed that the entheses of the masticatory muscles differ slightly from those of the trunk and limb muscles ${ }^{7}$. Specifically, the masticatory muscles contain three types of enthesis, including sites of direct tendon insertion into the bone. The authors concluded that these different types contribute to the unique bio-

Correspondence to: Dr. Satoru Matsunaga, Department of Anatomy, Tokyo Dental College, 2-9-18 Kandamisaki-cho, Chiyoda-ku, Tokyo 101-0061, Japan; Tel: +81-3-6380-9592; Fax: +81-3-6380-9664; E-mail: matsuna@tdc. ac.jp mechanical function of the masticatory muscles, enabling them to work as an "angle and stretching brake". However, the bones of the skull are subject to various functional pressures, including masticatory force, resulting in a complex relationship between bone structure and muscle function that remains to be fully elucidated. Clarification of the relationships between the micro- and nanostructural characteristics of the muscles, tendons, and bones in the maxillofacial area and the mechanical environment is required ${ }^{8,9)}$.

The relevance of bone quality in addition to bone density with regard to bone strength was proposed National Institutes of Health Consensus Development Conference in 2000. Since then, studies on the relationship between bone structural characteristics and bone strength have focused on bone quality factors ${ }^{10)}$. Collagen fibers and biological apatite (BAp) crystallites have been identified as dominant bone quality factors that are resistant to tensile and compressive stress, respectively, on bone tissue $\mathrm{e}^{11,12)}$.

Biological apatite is a hexagonal, ionic crystal that has a highly anisotropic nanostructure with preferential alignment along the $\mathrm{c}$ axis in the loading direction ${ }^{13)}$. Using microbeam X-ray diffraction analysis, $\mathrm{Na}-$ kano et al. quantitatively analyzed BAp crystallite alignment in animal trunk and limb bones, demonstrating a high correlation between 


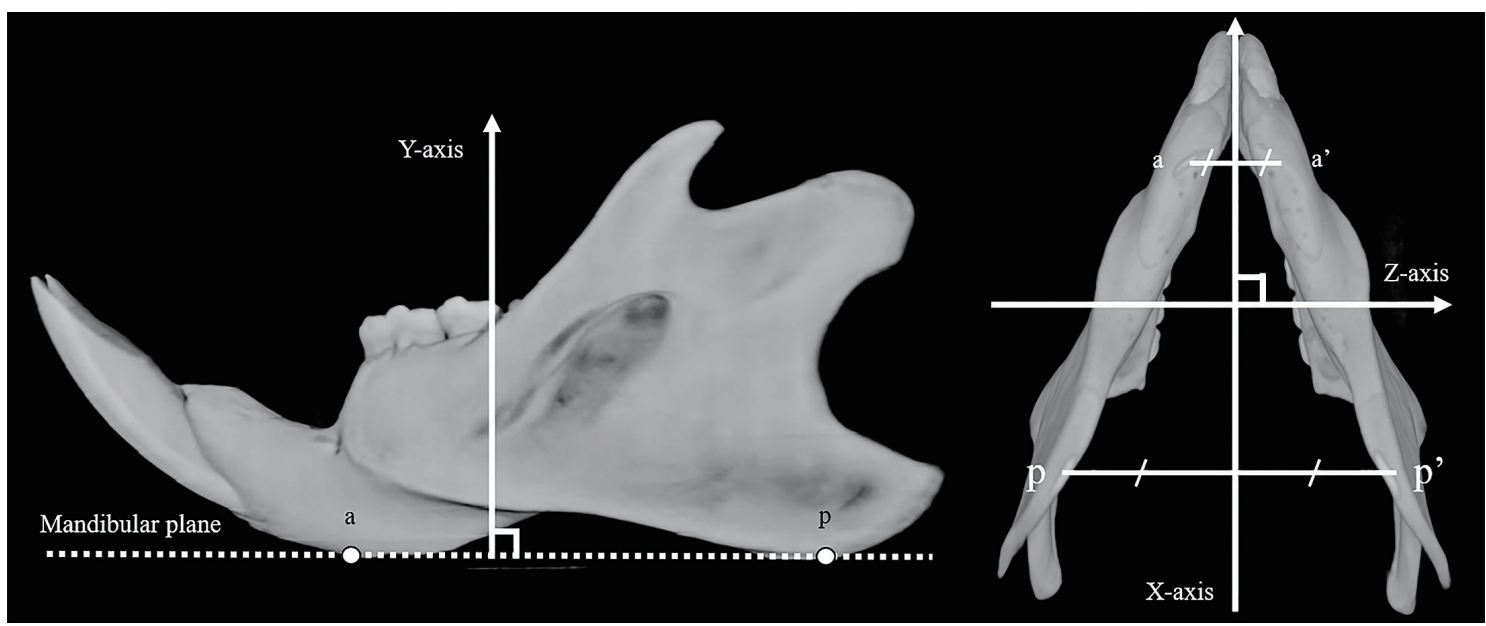

Figure 1. Reference points, plane and axes: point a; the lowest point in anterior thickening area of mandible. Point $\mathrm{p}$; the lowest point in posterior thickening area of mandible; Mandibular plane passing through a-a' and p-p' lines; X-axis passing through the mid-point of a-a' and p-p'; Y-axis the vertical axis against the mandibular plane; $\mathrm{Z}$-axis the vertical axis against the $\mathrm{X}-\mathrm{Y}$ plane.

mechanical stress and BAp crystallite alignment ${ }^{14,15)}$. With regard to collagen fibers as a bone quality factor, ongoing research by Vashishth et al. has shown that collagen crosslinks are a factor in age-related reductions in bone quality ${ }^{16)}$.

Meanwhile, Kawagoe et al. reported a relationship between bone strength and orientational anisotropy of collagen fibers for the masticatory muscles ${ }^{17)}$. Quantitative analysis of the jaw bone, particularly at entheses, should enable accurate prediction of the effects of the masseter muscles on the load environment of the mandible.

The present study aimed to clarify aspects of masseter muscle-tendon-bone morphological characteristics and local load environment through quantitative analysis of BAp crystallite alignment and collagen fiber orientation together with histological examination of the entheses.

\section{Specimens \\ Materials and Methods \\ The present study was approved by the Ethics Committee of Tokyo Dental College (Ethics Application No. 282807). Samples were pre- pared from the skulls of five 24-week-old male Wistar rats euthanized after deep anesthesia with ethyl ether.}

\section{Tissue slice preparation}

To obtain suitable samples for bone quality analysis, the left skull was embedded in autopolymerizing acrylic resin and sagittally sectioned using a saw microtome (SP1600; Leica Microsystems Inc., Wetzlar, Germany) with a blade width of $300 \mu \mathrm{m}$. Samples were then sanded using wet/dry sandpaper of increasing grit $(400,800$, and 1,200$)$ to prepare thin, $200 \mu \mathrm{m}$ slices. The right skull was fixed in $4 \%$ paraformaldehyde phosphate buffer solution and demineralized in $10 \%$ ethylenediaminetetraacetic acid (EDTA) for 4 weeks. Using standard methods, samples were embedded in paraffin embedding and sliced about $5 \mu \mathrm{m}$ thick in the coronal plane to enable observation of the masseter muscle entheses. Masson's trichrome staining were performed to observe the structural morphology of masseter muscle entheses in the first and third molar regions. And Toluidine blue staining were used to make the acidic mucus polysaccharide present in the cartilage metachromatic.

\section{Second harmonic generation (SHG) imaging}

SHG images were acquired using a multiphoton confocal microsco-

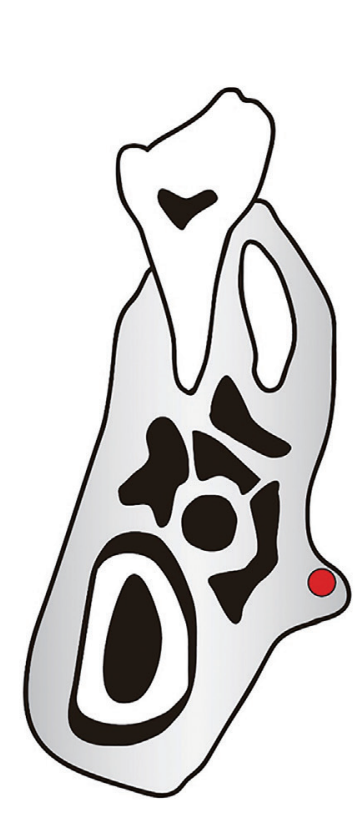

A

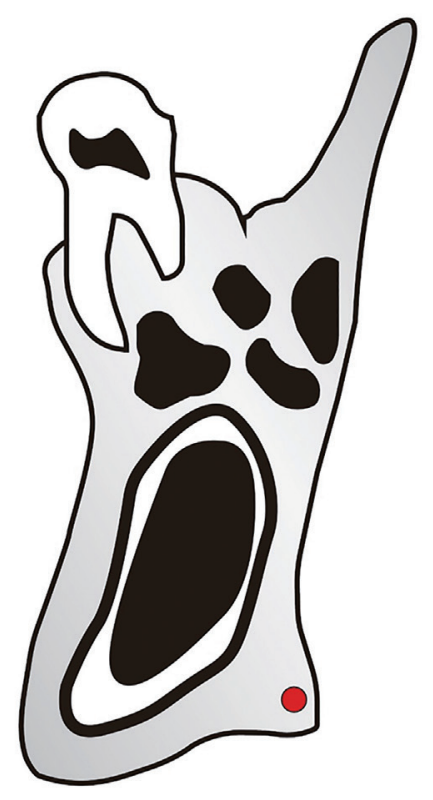

$\mathrm{B}$
Figure 2. Regions of interest in the mandible comprised masseter muscle entheses. The cortical bone of each red circle was selected in first molar region (A) and third molar region (B) and measurements were performed. Left; Lingual side. Right; Buccal side.

py system (A1R+MP, Nikon Instech Co., Ltd., Tokyo, Japan) with an excitation laser (Mai Tai eHP, wavelengths: 690-1,040 nm; repetition rate: $80 \mathrm{MHz}$; pulse width: $70 \mathrm{fs}$; Spectra-Physics, CA, US) and a water-immersion objective lens (CFI75 Apo 25×W MP, numerical aperture: 1.1; Nikon Instech Co., Ltd., Tokyo, Japan). The excitation wavelength for the observation of collagen fibers was $880 \mathrm{~nm}$. Image acquisition, processing for orthogonal views and cropping were performed using NIS-Elements version 4.0 (Nikon Instech Co., Ltd., Tokyo, Japan). Brightness and contrast of some images were adjusted using look-up tables (LUTs) of this software by the same parameters among relevant images to facilitate visibility. 

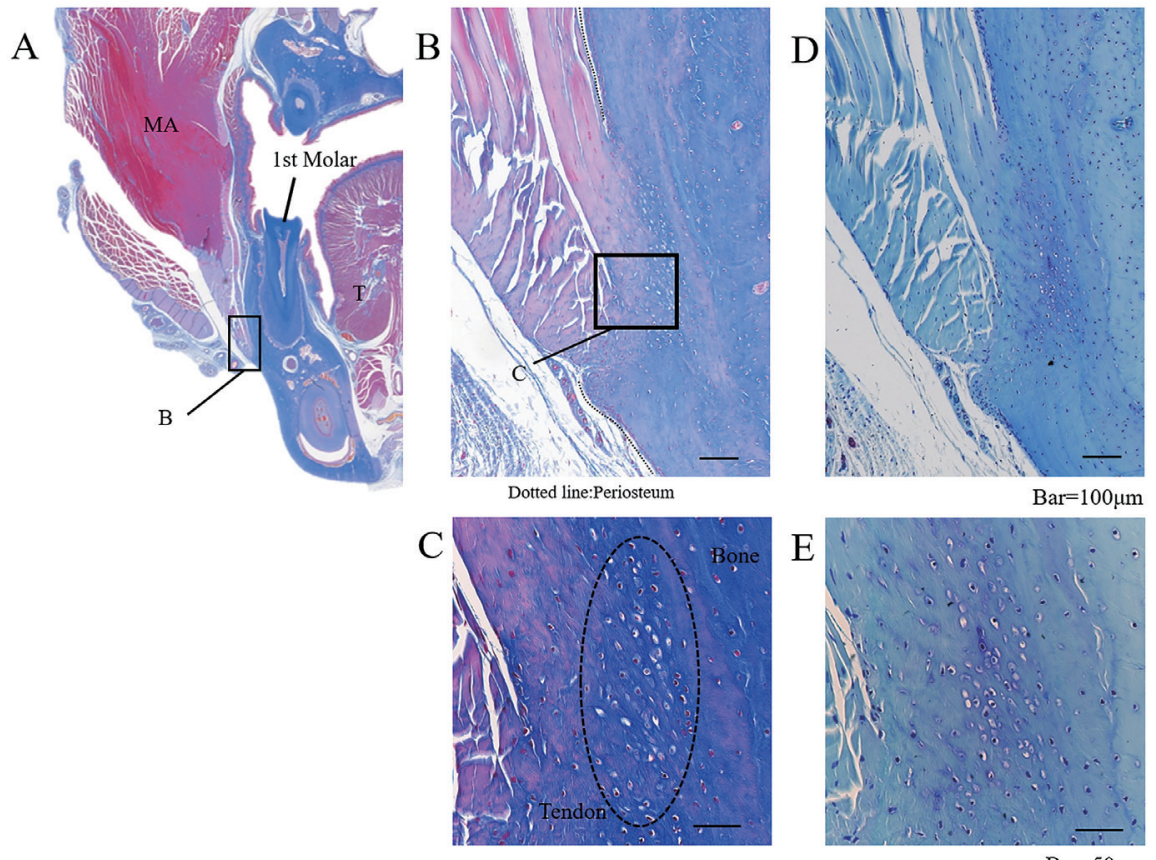

Figure 3. Masson's trichrome and toluidine blue staining of masseter muscle enthesis in the first molar region. (A) Whole image of the craniofacial area in coronal section. Masson's trichrome staining; magnification: $\times 25$. (B, D) The periosteum was fragmented and aggregation of chondrocytes was observed at the tendon-bone interface in the enthesis. The tendon was attached to the periosteum without rupturing. (B: Masson's trichrome staining. D: toluidine blue staining. magnification: $\times 100)(\mathrm{C}, \mathrm{E})$ Thickened chondrocytes (dotted line circle) were observed on the bone surface in the enthesis (C: Masson's trichrome staining. E: toluidine blue staining. Magnification: $\times 200)$ MA: masseter muscle. T: tongue. 1st Molar: first molar region.
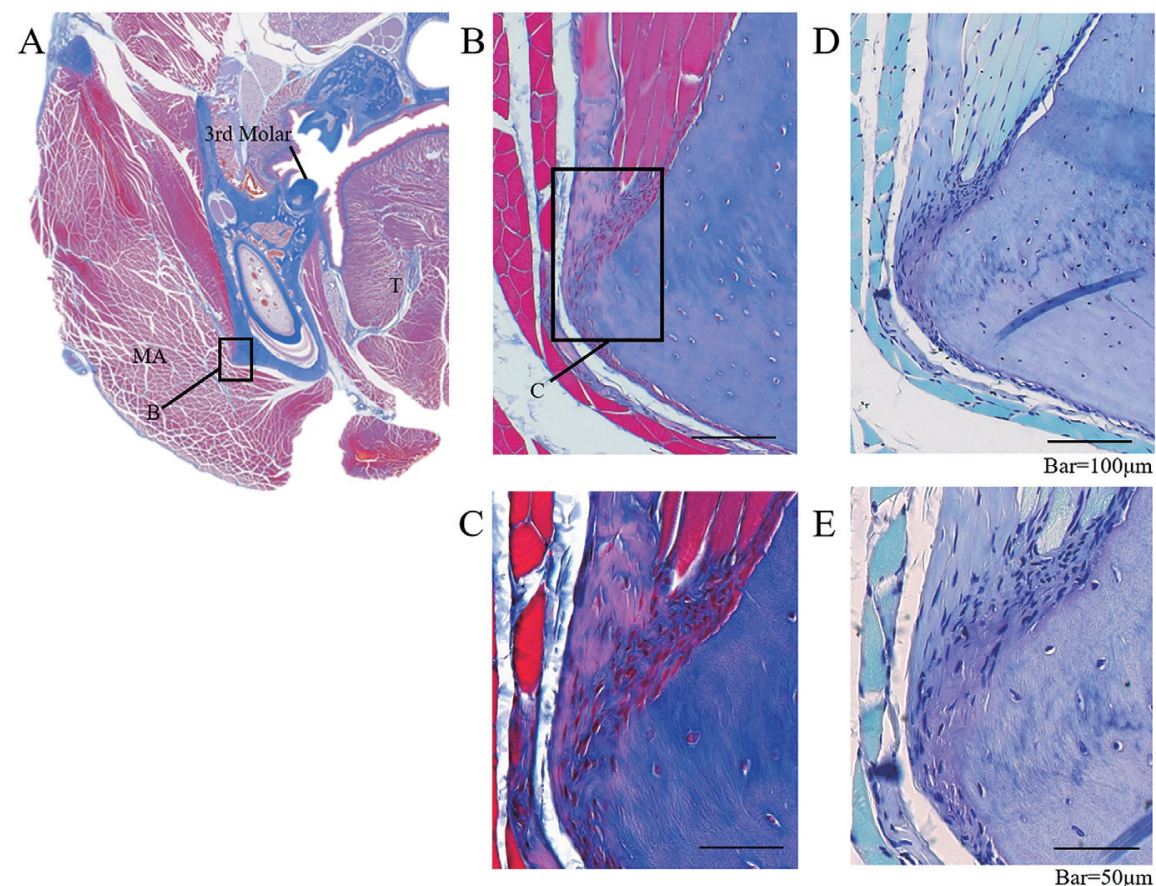

Figure 4. Masson's trichrome and toluidine blue staining of masseter muscle enthesis in the third molar region. (A) Muscle fibers could be seen covering the area from the lateral aspect to the base of the mandible, while thin tendons ran toward the cortical bone protuberance on the buccal side of the mandibular body. Masson's trichrome staining; magnification: $\times 25$. (B, D) The tendon of the masseter muscle was attached to the periosteum without rupturing. (B: Masson's trichrome staining. D: toluidine blue staining. Magnification: $\times 100)$. (C, E) The tendon thickened chondrocytes were observed on the bone surface in the enthesis. (C: Masson's trichrome staining. E: toluidine blue staining. Magnification: $\times 200$ ). MA: masseter muscle. T: tongue. 3rd Molar: third molar region. 


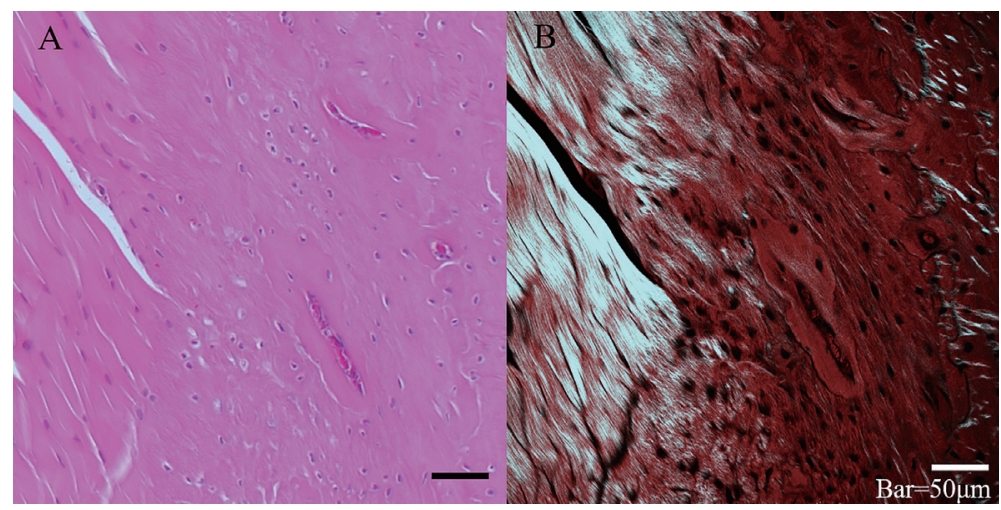

Figure 5. Coronal section in first molar region. (A) Hematoxylin and eosin staining. (B) Second harmonic generation imaging. The masseter muscle tendon in the first molar region comprised thick collagen fibers extending through the enthesis, some of which penetrated the cortical bone.

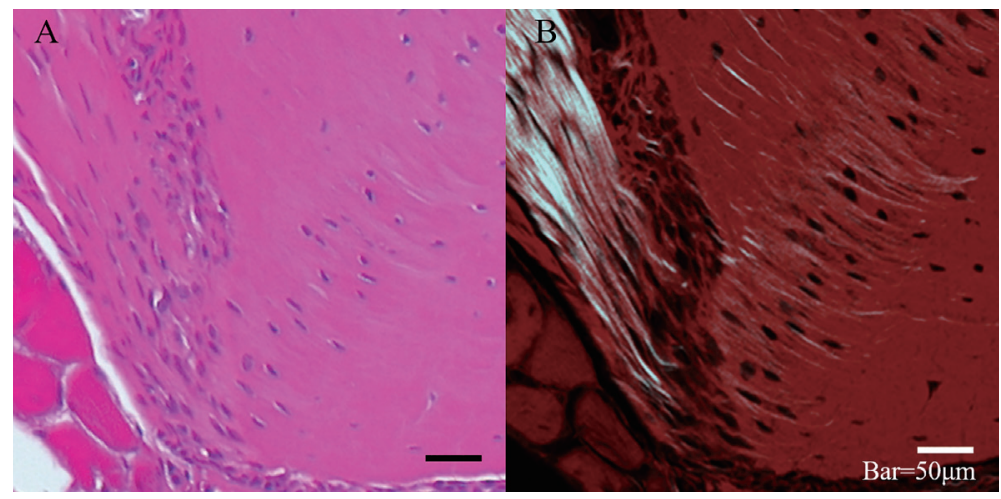

Figure 6. Coronal section in third molar region. (A) Hematoxylin and eosin staining. (B) Second harmonic generation imaging. In the vicinity of the enthesis, many collagen fibers inside the bone were observed running parallel to the orientation of the tendon.

A

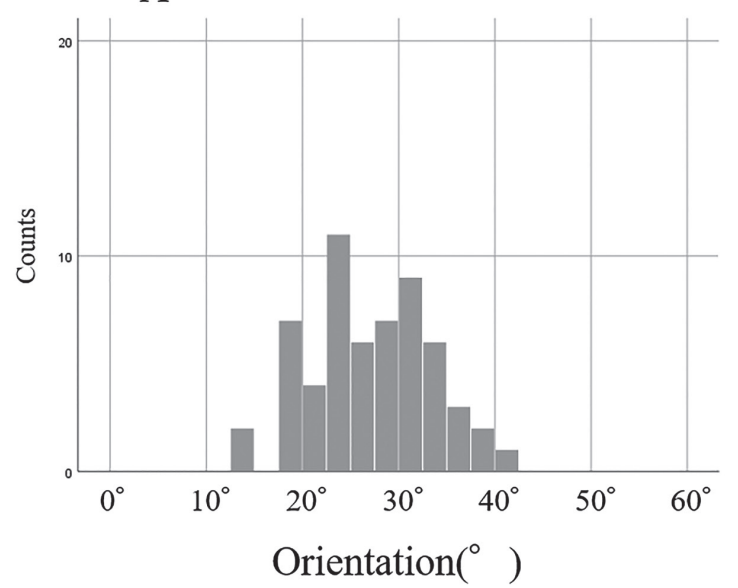

B

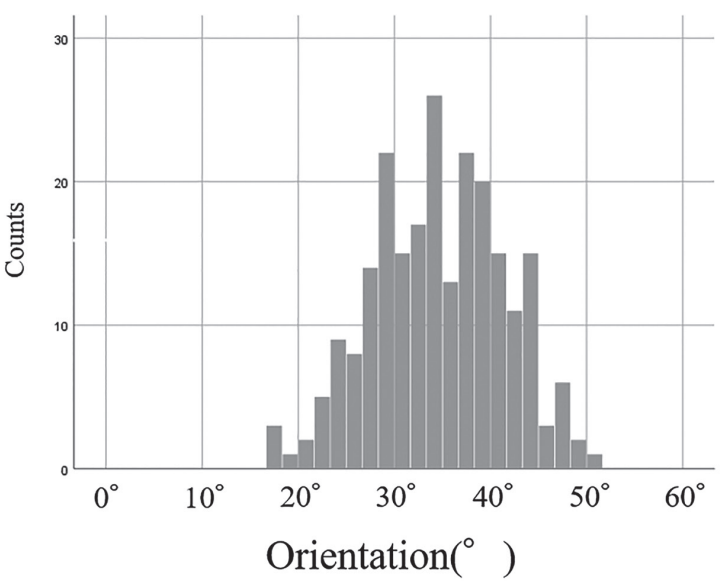

Figure 7. Orientation of tendon fibers in relation to bone. (A) First molar region. (B) Third molar region. In the first and third molar regions, tendon fibers ran tangentially to the bone at $31.1^{\circ}$ [standard deviation (SD), $\left.4.6^{\circ}\right]$ and $40.4^{\circ}\left[\mathrm{SD}, 3.5^{\circ}\right.$, respectively. 


\section{Micro-computed tomography (micro-CT) imaging}

Samples were examined using micro-computed tomography (micro-CT; HMX225 Actis4; Tesco Co., Tokyo, Japan) under the following imaging conditions: tube voltage, $140 \mathrm{kV}$; tube current, $100 \mu \mathrm{A}$; matrix size, $512 \times 512$; magnification, $\times 2.5$; slice width, $50 \mu \mathrm{m}$; and slice pitch, $50 \mu \mathrm{m}$. Three-dimensional reconstruction was performed using TRI/3DBON software (RATOC System Engineering Co., Ltd., Tokyo, Japan).

\section{BAp crystallite alignment}

Quantitative analysis of BAp crystallite alignment was conducted using an optical curved imaging plate (IP) X-ray diffraction system (XRD; D/MAX RAPID2-CMF; Rigaku Co., Tokyo, Japan). Measurements were performed in reflection and transmission modes with $\mathrm{Cu}-\mathrm{K \alpha}$ as the radiation source at a tube voltage of $40 \mathrm{kV}$ and tube current of 30 $\mathrm{mA}$. Reference axes were established in $\mathrm{X}$ axis, $\mathrm{Y}$ axis, and $\mathrm{Z}$ axis for each sample (Fig. 1) ${ }^{18,19)}$. Regions of interest in the mandible comprised masseter muscle entheses (Fig. 2). The radiation site was determined using the light microscope of the XRD system (magnification, $\times 0.6-4.8$ ), then an incident beam (diameter, $50 \mu \mathrm{m}$ ) was applied. Using reflection mode in the $\mathrm{X}$-axis direction and transmission mode in the Y-axis and $\mathrm{Z}$-axis directions, the diffracted $\mathrm{X}$-ray beam was detected using a curved IP based on the conditions described by Nakano et al. ${ }^{20)}$. The diffracted $\mathrm{X}$-ray beam was detected as a diffraction ring on the IP. Using 2-dimensional data-processing software (Rigaku Co., Tokyo, Japan), X-ray diffraction intensity ratios were calculated for the two diffraction peaks corresponding to planes 002 and 310.

\section{Statistical analysis}

Mean values for the five samples for each measurement point were calculated and compared using Tukey's multiple comparison test. Significance was set at $P<0.05$.

\section{Histological observation of entheses}

The results of Masson's trichrome staining and toluidine blue staining are showed of a coronal section in the first molar region of 24-weekold Wistar rat skulls (Fig. 3). Thick masseter muscle tendons with fibers largely grouped into bundles could be seen directly integrating into the buccal cortical bone. In the enthesis, the periosteum was fragmented and aggregation of chondrocytes was observed at the tendon-bone interface (Fig. 3B-E). The results of Masson's trichrome staining and toluidine blue staining are showed of a coronal section in the third molar region of 24-week-old Wistar rat skulls (Fig. 4).

In the masseter muscle enthesis, muscle fibers could be seen covering the area from the lateral aspect to the base of the mandible, while thin tendons ran toward the cortical bone protuberance on the buccal side of the mandibular body (Fig. 4A). The tendon was attached to the periosteum without rupturing (Fig. 4B, D) and thickened chondrocytes were observed on the bone surface in the enthesis (Fig. 4C, E).

\section{Orientational anisotropy of collagen fibers}

SHG images from the first molar region are showed in Fig. 5. The masseter muscle tendon in the first molar region comprised thick collagen fibers extending through the enthesis, some of which penetrated the cortical bone. As in the first molar region, collagen fibers in the third molar region ran toward the bone. However, these fibers were interrupted at the thickened periosteum. In the vicinity of the enthesis, many collagen fibers inside the bone were observed running parallel to the orientation of the tendon (Fig. 6). In the first and third molar regions, tendon

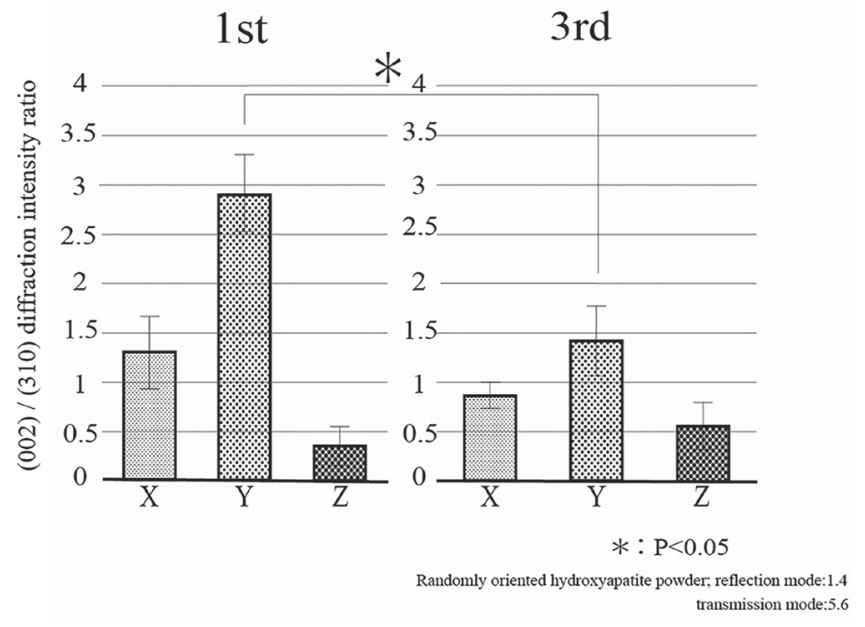

Figure 8. BAp crystallite alignment in masseter muscle enthuses. In both the first and third molar regions, strong uniaxial preferential alignment was noted in the $\mathrm{Y}$ axis direction in the masseter muscle entheses.

fibers ran tangentially to the bone at $31.1^{\circ}$ [standard deviation (SD), $\left.4.6^{\circ}\right]$ and $40.4^{\circ}\left[\mathrm{SD}, 3.5^{\circ}\right.$, respectively (Fig. 7 ).

\section{BAp crystallite alignment}

The angles of preferential alignment of BAp crystals are showed in an enthesis in relation to tooth axis in the first and third molar regions $\left(28^{\circ}\left[\mathrm{SD}, 10.95^{\circ}\right]\right)$ and $36^{\circ}\left[\mathrm{SD}, 8.94^{\circ}\right]$, respectively). X-ray diffraction intensity ratios calculated for the three reference axes for quantitative analysis are showed in Figure 8. The intensity ratios for hydroxyapatite powder were 1.4 and 5.6 in reflection and transmission modes, respectively. In both the first and third molar regions, strong uniaxial preferential alignment was noted in the Y-axis direction in the masseter muscle entheses. Furthermore, $\mathrm{X}$-ray diffraction intensity ratios in the Y-axis direction were significantly higher in the first compared to the third molar region.

\section{Discussion}

According to Huang et al., as the superior digital flexor tendon develops before and then joins with the subsequently formed digital bone, the related entheses penetrate the periosteum ${ }^{21)}$. Conversely, the entheses of many of the muscles in the trunk and limbs do not involve the periosteum, suggesting that these attachments are already formed at the stage of periosteal development ${ }^{22)}$. The present findings also show that, in the entheses of the masseter muscle in the first molar region, tendon attaches to bone via unmineralized fibrocartilage, while some tendon collagen fibers insert directly into the bone, running parallel to the muscle fibers. Furthermore, BAp crystallites in the same region show uniaxial preferential alignment at an angle that matches the insertion angle of the tendon fibers. This suggests that both the anisotropy of the collagen fibers and BAp crystallite alignment confer high resistance in the direction of the masseter muscle tendon. As the masseter muscle in the first molar region is directly attached to the bone via the tendon, these structural characteristics may optimize bone quality to enable the high load generated by muscle contraction to be efficiently transmitted from tendon to bone ${ }^{23)}$.

Conversely, in the entheses of the masseter muscle in the third molar region, the tendon attaches to the bone via a layer of thickened periosteum and chondrocytes. Muscles with entheses that indirectly attach to the bone via the periosteum do not produce large functional pressures; 
rather, they are responsible for precise movements ${ }^{24)}$. As in the first molar region, the results of bone quality analysis in the third molar region showed BAp crystallite alignment parallel to the orientation of the tendon fibers. However, the intensity ratio values were significantly lower. This indicates that the local mechanical environment generates differences in enthesis morphology.

Matsumoto et al. analyzed bone quality in human jaw bones and found uniaxial preferential alignment of BAp crystallites and high bone strength in the tooth axis direction in specific alveolar bone ${ }^{25}$. Meanwhile, Nakano et al. demonstrated a strong positive correlation between bone quality factors and bone strength, indicating that the mechanical environment of entheses determines the orientation of the collagen fibers, which is linked to the preferential alignment of BAp crystallites. Changing the amount and direction of functional pressure from the muscles may thus affect not only bone density, but also bone density.

\section{Acknowledgements}

This study was supported by a research grant from the Japan Society for the Promotion of Science; contract grant number: 18K09643, $17 \mathrm{~K} 11808$ and was partly supported by a grant of Multidisciplinary Research Center for Jaw Disease (MRCJD): Achieving Longevity and Sustainability by Comprehensive Reconstruction of Oral and Maxillofacial Functions.

\section{Conflict of Interest}

The authors have no COI exists.

\section{References}

1. Benjamin M, Evans EJ and Copp L. The histology of tendon attachments to bone in man. J Anat 149: 89-100, 1986

2. Holger $\mathrm{P}$ and Martin S. Histological evidence for muscle insertion in extant amniote femora: implications for muscle reconstruction in fossils. J Anat 222: 419-436, 2013

3. Evans E J, Benjamin M and Pemberton DJ. Variations in the amount of calcified tissue at the attachments of the quadriceps tendon and patellar ligament in man. J Anat 174: 145-151, 1991

4. Apostolakos J, Durant TJ, Dwyer CR, Russell RP, Weinreb JH, Alaee F, Beitzel K, McCarthy MB, Cote MP and Mazzocca AD. The enthesis: a review of the tendon-to-bone insertion. Muscles Ligaments Tendons J 4: 33-42, 2014

5. Thomopoulos S, Williams GR, Gimbel JA, Favata M and Soslowsky LJ. Variations of biomechanical, structural, and compositional properties along the tendon to bone insertion site. J Orthop Res 1: 13-19, 2003

6. Schwartz AG, Lipner JH, Pasteris JD, Genin GM and Thomopoulos $\mathrm{S}$. Muscle loading is necessary for the formation of a functional tendon enthesis. Bone 55: 44-51, 2013

7. Hems T and Tillmann B. Tendon entheses of the human masticatory muscles. Anat Embryol (Berl) 202: 201-208, 2000

8. Yamada K. Histological study on the migration mechanism of the attachment of the deep layer of the masseter muscle to the rat mandible during growth. Jpn J Oral Biol 27: 254-271, 1985
9. Doreen A and Carla A. Histologic study of the attachment of muscle to the rat mandible. Arch Oral Biol 27: 519-527, 1982

10. NIH consensus development panel on osteoporosis prevention, diagnosis, and therapy. Osteoporosis prevention, diagnosis, and therapy. JAMA 285, 785-795, 2001

11. Sasaki N and Sudoh Y. X-ray pole figure analysis of apatite crystals and collagen molecules in bone. Calcif Tissue Int 60: 361-367, 1997

12. Wang $J$, Ishimoto $T$ and Nakano T. Unloading-induced degradation of the anisotropic arrangement of collagen/apatite in rat femurs. Calcif Tissue Int 100: 87-94, 2017

13. Elliot JC. Structure and Chemistry of the Apatites and Other Calcium Orthophosphates. Amsterdam: Elsevier Science; 1994

14. Jimbo R, Nakano T and Sawase T. Alignment of biological apatite c-axis under functional loading:a preliminary report. Implant Dent 25: 594-598, 2016

15. Nakano T, Kaibara K, Ishimoto T, Tabata Y and Umakoshi Y. Biological apatite (BAp) crystallographic orientation and texture as a new index for assessing the microstructure and function of bone regenerated by tissue engineering. Bone 51: 741-747, 2012

16. Vashishth D. The role of the collagen matrix in skeletal fragility. Curr Osteoporos Rep 5: 62-66, 2007

17. Benjamin M, Kumai T, Milz S, Boszczyk BM, Boszczyk AA and Ralphs JR. The skeletal attachment of tendons--tendon "entheses". Comp Biochem Physiol A Mol Integr Physiol 133: 931-945, 2002

18. Xiao F, Hayashi H, Fujita T,Shirakura M, Tsuka Y, Fujii E, Tanne K and Tanimoto K. Role of articular disc in cartilaginous growth of the mandible in rats. APOC 7: 29-34, 2017

19. Fujita T, Ohtani J, Shigekawa M, Kawata T, Kaku M, Kohno S, Tsutsui K, Tenjo K, Motokawa M, Tohma Y and Tanne K. Effects of Sex Hormone Disturbances on Craniofacial Growth in Newborn Mice. J Dent Res. 83: 250-254. 2004

20. Nakano T, Kaibara K, Tabata Y, Nagata N, Enomoto S, Marukawa E and Umakoshi Y. Unique alignment and texture of biological apatite crystallites in typical calcified tissues analyzed by microbeam X-ray diffractometer system. Bone 31: 479-487, 2002

21. Huang AH, Riordan TJ, Wang L, Eyal S, Zelzer E, Brigande JV and Schweitzer R. Repositioning forelimb superficialis muscles: tendon attachment and muscle activity enable active relocation of functional myofibers. Dev Cell 26: 544-551, 2013

22. Berendsen AD and Olsen BR. Bone development. Bone 80: 14-18, 2015

23. Apostolakos J, Durant TJ, Dwyer CR, Russell RP, Weinreb JH, Alaee F, Beitzel K, McCarthy MB, Cote MP and Mazzocca AD. The enthesis: a review of the tendon-to-bone insertion. Muscles Ligaments Tendons J 4: 333-342, 2014

24. Lu HH and Thomopoulos S. Functional attachment of soft tissues to bone: development, healing, and tissue engineering. Annu Rev Biomed Eng 15: 201-226, 2013

25. Matsumoto T, Matsunaga S, Morioka T, Nakano T, Yoshinari M and Yajima Y. Relationship between preferential alignment of biological apatite and Young's modulus at first molar in human mandible cortical bone. J Hard Tissue Biol 22: 163-170, 2013 